\title{
What Are All the Best Sphere Packings in Low Dimensions?
}

\author{
J. H. Conway ${ }^{1}$ and N. J. A. Sloane ${ }^{2}$ \\ ${ }^{1}$ Mathematics Department, Princeton University, \\ Princeton, NJ 08544, USA \\ ${ }^{2}$ Mathematical Sciences Research Center, AT \& T Bell Laboratories, \\ Murray Hill, NJ 07974, USA
}

\begin{abstract}
We describe what may be all the best packings of nonoverlapping equal spheres in dimensions $n \leq 10$, where "best" means both having the highest density and not permitting any local improvement. For example, the best five-dimensional sphere packings are parametrized by the 4-colorings of the one-dimensional integer lattice. We also find what we believe to be the exact numbers of "uniform" packings among these, that is, those in which the automorphism group acts transitively. These assertions depend on certain plausible but as yet unproved postulates.

Our work may be regarded as a continuation of László Fejes Tóth's work on solid packings.
\end{abstract}

\section{Introduction}

Stated informally, the object of this paper is to describe what may be all the best (that is, "tightest") packings of nonoverlapping equal spheres in up to 10 dimensions. In Propositions 1-10 we give a very precise description of these packings.

There are some surprises. We show that the Korkine-Zolotarev lattice $\Lambda_{9}$ (which continues to hold the density record it established in 1873) has the following astonishing property. Half the spheres can be moved bodily through arbitrarily large distances without overlapping the the other half, only touching them at isolated instants, and yet the packing apparently remains tight at all times. We also discuss some new higher-dimensional packings, showing for example that there are extraordinarily many 16-dimensional packings that are just as dense as the Barnes-Wall lattice $\Lambda_{16}$.

However, although the answers are precise, the question we are answering is not so easily formulated, and there is a second difficulty in that the results depend on 
certain as yet unproved "postulates." In short, we face great difficulties both in asking the question of our title in a precise way, and also in answering it. For these two reasons we have delayed publication of this work for some time. Nevertheless, these packings are so interesting that we feel they should be placed on record. We hope that this will stimulate further research on these problems.

The first difficulty arises when we attempt to give a precise definition of the class of packings we are characterizing, that is, to answer the question: what does it mean to be one of the "best" packings? Certainly they should have the highest density, a well-defined concept [Ro]. However, there are many examples which show that one should not be interested in every packing with the highest density. For example, if a finite number of spheres are removed from a packing then its density is unchanged. Nor is the density changed if we increase the radius vector from the origin to each center by its arctangent, although this change removes all contacts between the spheres. In neither case are the new packings "tight." Many other examples could be given.

It seems difficult to give a precise definition of this concept of "tightness." Professor Fejes Tóth [FT2] (see also [FT1]) has discussed such problems and has introduced the notion of a "solid" packing to solve some of them. A packing is solid if no finite subset of the spheres can be rearranged so as to form, together with the rest of the spheres, a packing not congruent to the original one. A solid packing necessarily has the highest density.

However, even a solid packing can still contain quite large cavities, and we need a further notion. The flaws in the packings mentioned above suggest the following definition.

Consider the set of centers of the spheres, which from now on we shall identify with the packing. Suppose we can dissect the space of the packing into finitely many polyhedral pieces (possibly infinite) in such a way that each center lies in the interior of some piece, and there are also some empty pieces containing no centers. Then if we can rearrange the nonempty pieces into another dissection in which the centers are at least as far apart as they were originally, we call the packing loose.

Provisionally, we may call a packing tight if it is not loose. Packings that are tight in this sense certainly have the highest possible density. However, we are not sure that this particular definition is the right one, and perhaps some other meaning for "tight" should be used in the postulates below.

The goal of this paper is to describe all the tight packings in dimensions $n \leq 10$, and to give partial information about some higher dimensions.

After we have assigned a provisional meaning to the question of our title, we come to a second difficulty, that of answering it!

Since at present the highest density of a sphere packing in $\mathbb{R}^{n}$ is known only for $n=0,1$, and 2 (in spite of much recent work [Ha1], [Ha2], [Hs], [Mu] on the case $n=3$ ), we cannot hope to make any absolute assertions about tight packings in higher dimensions. Instead we base our results on certain (as yet unproved) "postulates."

We say that an $n$-dimensional packing $P_{n}$ fibers over an $m$-dimensional packing $P_{m}$ if $P_{n}$ can be decomposed into sets (or layers) of points lying in parallel $m$-dimensional spaces, each one of which is a packing of type $P_{m}$.

Our $n$th (for $n \geq 2$ ) postulate is the following. 
Postulate $n$. Any tight $n$-dimensional packing fibers over some tight $2^{k}$-dimensional packing, where $2^{k}$ is the largest power of 2 strictly less than $n$.

We believe (but cannot prove) that Postulate $n$ is true for $2 \leq n \leq 8$. Postulate 9 requires modification, and Postulate 10 is irredeemably false (see Sections 9 and 10).

The main results of this paper are given in Propositions 1-10, where Proposition $n$ (which depends on some of our postulates) describes all possible tight packings in dimension $n$. We know much less about higher dimensions, and just describe a few more packings that seem likely to be tight.

For $n \leq 8$ we also describe all the uniform tight packings, those in which the automorphism group acts transitively on the spheres. The putative number of uniform tight packings in each dimension is given by the following table:

\begin{tabular}{llllllllllll}
\hline$n$ & 0 & 1 & 2 & 3 & 4 & 5 & 6 & 7 & 8 & 9 & 10 \\
Number & 1 & 1 & 1 & 2 & 1 & 4 & 4 & 4 & 1 & $\infty$ & 1 \\
\hline
\end{tabular}

The uniform packings in five dimensions were found by Leech [Le1]. He also found some of the six- and seven-dimensional ones [Le2].

The densest possible lattice packings are known in dimensions $0 \leq n \leq 8$. They are the root lattices

$$
A_{0}, A_{1}, A_{2}, A_{3}=D_{3}, D_{4}, D_{5}=E_{5}, E_{6}, E_{7}, E_{8}
$$

(see [CS1], which we use as our general reference for information about lattices and other sphere packings). It is conjectured that the highest densities in dimensions $n \leq 8$ are attained by these lattices, and in nine dimensions by the "laminated" lattice $\Lambda_{9}$. These records were already known to Korkine and Zolotareff [KZ] in 1873. However, in the next few dimensions the records have been improved several times. The current record densities for lattices in dimensions 10-13 are held by

$$
\Lambda_{10}, \mathscr{K}_{11}, \mathscr{K}_{12}, \text { and } \mathscr{K}_{13},
$$

respectively discovered by Chaundy [Ch], Barnes [Ba2], Coxeter and Todd [CT], and Conway [Co], but in 10,11, and 13 dimensions there are better nonlattice packings:

$$
P_{10 c}, P_{11 a}, \text { and } P_{13 a}
$$

respectively discovered by Best [Be], and Leech and Sloane [LS1], [LS2].

The minimal nonzero norm (or squared length) in the standard definitions of the above root lattices is 2 , and we accordingly fix the radius of the spheres in all our packings at $1 / \sqrt{2}$.

The following notation in used throughout. $\Pi_{n}(n \geq 2)$ denotes a densest $n$ dimensional packing that fibers over a tight $2^{k}$-dimensional packing, where $2^{k}$ is the highest power of 2 strictly less than $n$. For $2 \leq n \leq 8$, Postulate $n$ implies that tight packings have this form. We also define $\Pi_{n}$ to be a tight $n$-dimensional packing for $n=0,1$. We use the names $\Lambda_{n}^{1}, \Lambda_{n}^{2}, \ldots$ for the most interesting particular cases, since these are analogues of the laminated lattices $\Lambda_{n}=\Lambda_{n}^{1}$ of Chapter 6 of [CS1].

Suppose $P_{n}$ is an $n$-dimensional packing that fibers over an $m$-dimensional packing $P_{m}$. Let $A$ be the projection onto the space of a layer $P_{m}$ of a point in another layer $P_{m}^{\prime}$. Let $x$ be the distance of $A$ from the closest point of $P_{m}$, and let $y$ 

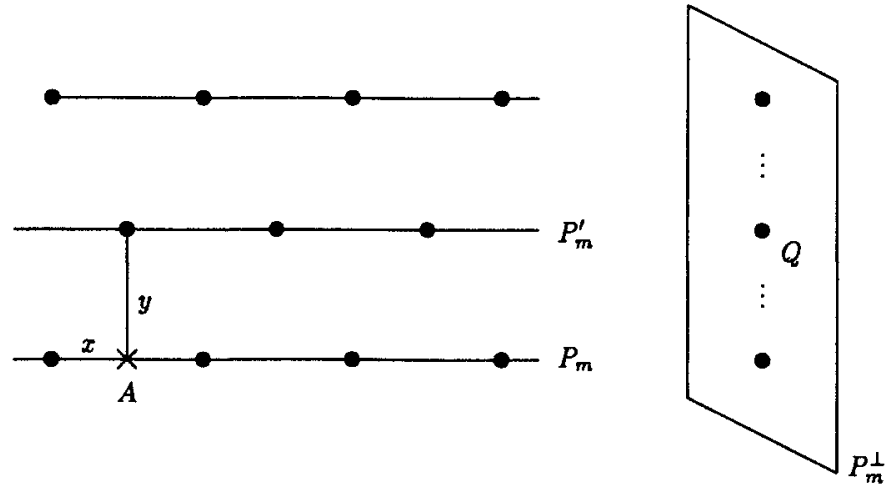

Fig. 1. A fibered packing and its quotient $Q$.

be the separation between the layers $P_{m}$ and $P_{m}^{\prime}$ (Fig. 1). Then $x$ is at most the covering radius $R$ of $P_{m}$, and $x^{2}+y^{2} \geq 2$, so $y$ is at least $\sqrt{2-R^{2}}$.

Let $Q$ be the projection of $P_{n}$ onto the space $P_{m}^{\perp}$. If we surround each point of $Q$ by a sphere of diameter $\sqrt{2-R^{2}}$, we obtain a sphere packing in $P_{m}^{\perp}$. If $P_{n}$ is to be tight, it is plainly necessary that this sphere packing in $P_{m}^{\perp}$ should be tight, and that $P_{m}$ also be tight. (Our Postulate $n$ asserts that all the tight $n$-dimensional packings can be obtained in this way, taking $m=2^{k}$.) This can only happen if for adjacent layers $P_{m}$ and $P_{m}^{\prime}$ it always happens that every point of $P_{m}^{\prime}$ lies above a deep hole of $P_{m}$.

\section{Dimension 1}

Our first assertion needs no proof (note that our spheres have radius $1 / \sqrt{2}$, and the minimal squared distance between distinct points of our packings is 2 ).

Proposition 1. The only tight packing $\Pi_{1}$ is the root lattice $\Lambda_{1}=A_{1}$.

We recall that $A_{1}=\langle(1,-1)\rangle$, i.e., is generated by the vector $(1,-1)$ [CS1]. The dual lattice $A_{1}^{*}$ is the union of two cosets [0] $=A_{1}$ and [1] $=A_{1}+\left(\frac{1}{2},-\frac{1}{2}\right)$ (Fig. 2). Each of the cosets [0], [1] consists of the deep holes in the other. The covering radius of $A_{1}$ is $1 / \sqrt{2}$.

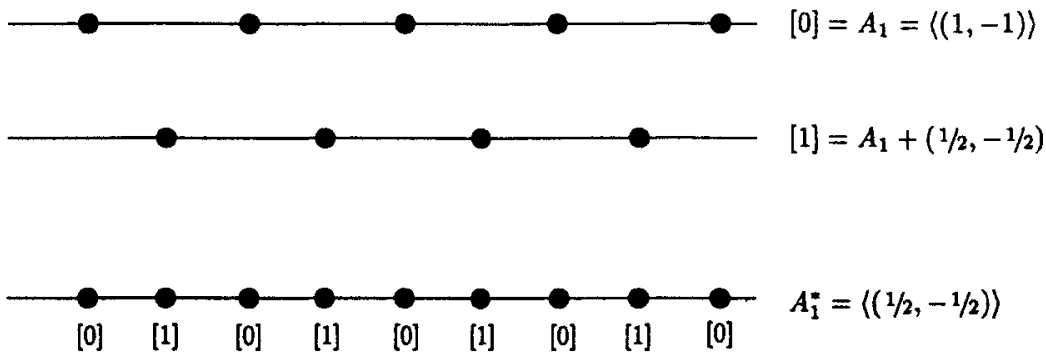

Fig. 2 


\section{Dimension 2}

Proposition 2 (depends on Postulate 2). The only tight packing $\mathrm{I}_{2}$ is the root lattice $\Lambda_{2}=A_{2}$.

Proof. Postulate 2 tells us that $\Pi_{2}$ fibers over $A_{1}$. From the discussion at the end of the Introduction, the separation between the $A_{1}$ layers is at least $\sqrt{2-\frac{1}{2}}=\sqrt{\frac{3}{2}}$, and this can be achieved only for the packing shown in Fig. 3, in which alternate layers are the cosets [0] and [1] of $A_{1}$ in $A_{1}^{*}$. The packing $Q$ is a rescaled $A_{1}$ in which the points are colored alternately [0] and [1]. What results is of course a description of the root lattice $A_{2}$.

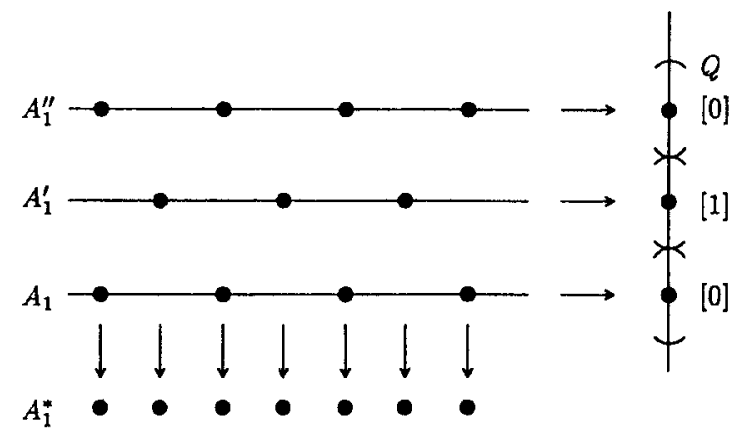

Fig. 3

We shall introduce a language for describing fibered packings by reference to this case. Each layer here projects onto the starting layer, either onto [0] or onto [1]. We call the possible projections placements. The placements of adjacent layers must be adjacent in the placement graph, which in this case is the graph $2_{1}$ consisting of a single edge:

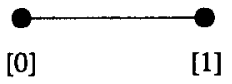

In general, the layers in a tight packing can have only a limited number of projections onto the starting layer: these are the placements. Two placements are joined by an edge in the placement graph just if they can correspond to layers at the minimal distance $\sqrt{2-R^{2}}$, that is, if each point of either projects onto a deep hole in the other. We use $v_{k}$ to denote a graph with $v$ vertices and valence $k$.

Fibering over $A_{2}$. The lattice $A_{2}^{*}$ dual to $A_{2}$ consists of the three cosets

$$
[0]=A_{2}, \quad[1]=A_{2}+\left(\frac{2}{3},\left(-\frac{1}{3}\right)^{2}\right), \quad[2]=A_{2}+\left(-\frac{2}{3},\left(\frac{1}{3}\right)^{2}\right)
$$


of $A_{2}$. The deep holes in any one of these cosets are the union of the other two cosets, and the covering radius is $\sqrt{\frac{2}{3}}$. Each of the cosets [0], [1], [2], and also their union $A_{2}^{*}$, is a version of the regular hexagonal lattice (see Fig. 4).

$\begin{array}{llllllll}{[0]} & & {[0]} & & {[0]} & & {[0]} & \\ & {[1]} & & {[1]} & & {[1]} & & \\ & & {[2]} & & {[2]} & & {[2]} & \\ & {[0]} & & {[0]} & & {[0]} & & {[0]} \\ & & {[1]} & & {[1]} & & {[1]} & \\ & {[2]} & & {[2]} & & {[2]} & & \\ {[0]} & & {[0]} & & {[0]} & & {[0]} & \end{array}$

Fig. 4

\section{Dimension 3}

Proposition 3 (depends on Postulates 2 and 3). The tight packings $\Pi_{3}$ are parametrized by 3-colorings of the one-dimensional packing $A_{1}$, or alternatively by random walks on the triangle $3_{2}$.

We call these the Barlow packings, since Barlow [Ba1] studied some of them in 1883 .

Proof. Postulates 2 and 3 tell us that each layer in a $\Pi_{3}$ is a copy of an $A_{2}$ and that the separation between any two layers is at least $\sqrt{2-\frac{2}{3}}=\sqrt{\frac{4}{3}}$. Now suppose adjacent layers are always at this distance, and choose a particular layer which we take to be $A_{2}=[0]$. Then the next layer must be a copy of $A_{2}$ contained in [1] $\cup$ [2]. However, since squared distances in $A_{2}$ are even integers, the next layer cannot contain points from both [1] and [2] (the squared distances between which have the form $2 n+\frac{2}{3}$ ), so must be all of [1] or [2], say [1]. The layer after this must now be [0] or [2], and so on. We conclude that every layer of a $\Pi_{3}$ lies above one of the three cosets [0], [1], [2] of $A_{2}$ in $A_{2}^{*}$, adjacent layers lying above different cosets. In our formal language we express this by saying that there are three placements of $A_{2}$, and the placement graph is a triangle $3_{2}$ :

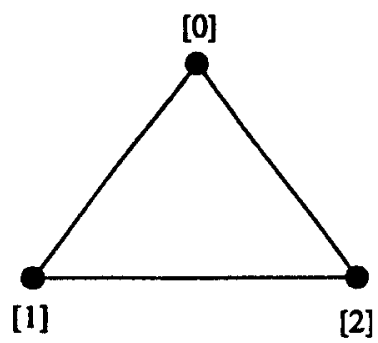

So indeed the tight packings are obtained from 3-colorings of $A_{1}$. 
Uniform Packings. A sphere in such a Barlow packing can be surrounded in two essentially different ways. If the two neighboring layers come from different cosets of $A_{2}$, the contact points of the neighboring spheres form a cuboctahedron [CR, Fig. 101]; if from the same coset, a pseudocuboctahedron (obtained by placing a cuboctahedron so a triangular face is uppermost and the "equator" is a hexagon, and replacing the bottom half by the reflection of the top half in the equatorial plane). So there are only two choices for a uniform packing $\Pi_{3}$, namely, $\Lambda_{3}^{1}=\Lambda_{3}=$ $A_{3}$, the face-centered cubic (or f.c.c.) lattice, in which successive layers are

$$
\Lambda_{3}^{1}: \quad \cdots \quad 0 \quad 1 \quad 2 \quad 0 \quad 122 \quad \cdots
$$

(we omit the square brackets to save space) and $\Lambda_{3}^{2}$, the hexagonal close packing (or h.c.p.), in which they are

$$
\Lambda_{3}^{2}: \quad \cdots \quad 122112012
$$

Crystal Balls. We define the first crystal ball of a sphere in a packing to consist of that sphere and those touching it; and inductively, the $(n+1)$ st crystal ball to consist of the $n$th crystal ball together with all the spheres that touch it.

In all the Barlow packings a sphere has 12 neighbors, so the first crystal ball has size 13 . However, the second crystal ball can have size 55,56 , or $57 .{ }^{1}$ Consider five adjacent layers colored $a, b, c, d, e$, where each color is one of [0],[1], [2]. Then the second crystal ball for a sphere in layer $c$ has size $55+$ number of "Yes" answers to the two questions:

$$
\text { Is } a=c \text { ? } \quad \text { Is } c=e \text { ? }
$$

(Fig. 5 shows a case in which $a=c, c \neq e$.)

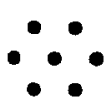

7

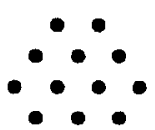

12

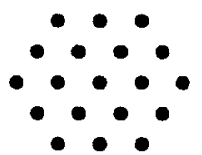

19

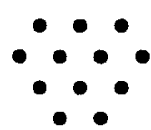

12

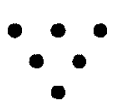

$6=$ 56

Fig. 5. How the 56 spheres of a second crystal ball are distributed into five layers in a typical Barlow packing.

There are just four packings that are characterized by the set of sizes of their second crystal balls. If no such ball has size 56, then the answers to alternate questions from the sequence:

$$
\text { ‥ Is } a=c ? \quad \text { Is } b=d ? \quad \text { Is } c=e ? \quad \ldots
$$

\footnotetext{
${ }^{1}$ It is asserted on p. 801 of [Hs] that the second crystal balls in all the Barlow packings have size 57 , but as we shall see this is incorrect.
} 
must always be the same, and we obtain one of the three packings:

$\begin{array}{cccc}\text { Packing } & \text { Answers } & \text { Coloring } & \text { Sizes } \\ \Lambda_{3}^{1} & \cdots \text { NNNN } \cdots & \cdots 012012012 \cdots & 55 \\ \Lambda_{3}^{2} & \cdots \text { YYYY } \cdots & \cdots 01010101 \cdots & 57 \\ \Lambda_{3}^{3} & \cdots \text { YNYN } \cdots & \cdots 0102010201 \cdots & 55,57\end{array}$

If all the second crystal balls have size 56, then alternate answers must always be different, and we find a fourth packing:

$$
\Lambda_{3}^{4} \quad \cdots \text { YYNNYYNN } \cdots \quad \cdots 020210102121 \cdots \quad 56 .
$$

We have already mentioned $\Lambda_{3}^{1}$ and $\Lambda_{3}^{2}$ in the preceding subsection. $\Lambda_{3}^{3}$ and $\Lambda_{3}^{4}$ are not uniform, the nonuniformity of $\Lambda_{3}^{4}$ being first revealed by the fact that its third crystal balls are not of constant size.

For each of the remaining three sets $\{55,56\},\{56,57\},\{55,56,57\}$ it is easy to see that there are uncountably many packings for which these are the sets of second crystal ball numbers.

The spheres in $\Lambda_{3}^{3}$ and $\Lambda_{3}^{4}$ lie in just two orbits under the automorphism groups of these packings. Such packings were studied by Melmore [Me]. There are precisely two further packings with this property:

$$
\begin{array}{llll}
\Lambda_{3}^{5} & \cdots \text { YYNYYN } \cdots & \cdots 010202121010 \cdots & 56,57, \\
\Lambda^{6} & \cdots \text { YNNYNN } \cdots & \cdots 012021012021 \cdots & 55,56 .
\end{array}
$$

Note that there is a packing $\Lambda_{3}^{n}(n \geq 6)$ corresponding to each nonempty proper subset of $\{55,56,57\}$. For $n=1,2,3,4,5,6$ respectively, the packing $\Lambda_{3}^{n}$ consists of $1,2,4,4,3,6$ translates of a lattice packing.

\section{Dimension 4}

Proposition 4 (depends on Postulates 2 and 4). The only tight packing $\Pi_{4}$ is the root lattice $\Lambda_{4}=D_{4}$.

Proof. Here both the layers and the quotient space are two-dimensional (Fig. 6), and any tight packing must come from a 3-coloring of the two-dimensional packing $Q$, which is a rescaled version of $A_{2}$. However, this is unique (Fig. 7), since it is determined by the colors of three adjacent circles. The resulting lattice is $D_{4}$.

The dual lattice $D_{4}^{*}$ consists of four cosets,

$$
\begin{gathered}
{[0]=D_{4}, \quad[1]=D_{4}+\left(\frac{1}{2}, \frac{1}{2}, \frac{1}{2}, \frac{1}{2}\right), \quad[2]=D_{4}+(0,0,0,1),} \\
{[3]=D_{4}+\left(\frac{1}{2}, \frac{1}{2}, \frac{1}{2},-\frac{1}{2}\right),}
\end{gathered}
$$

and the set of deep holes in any one of these cosets is the union of the other three. The covering radus is 1 . 


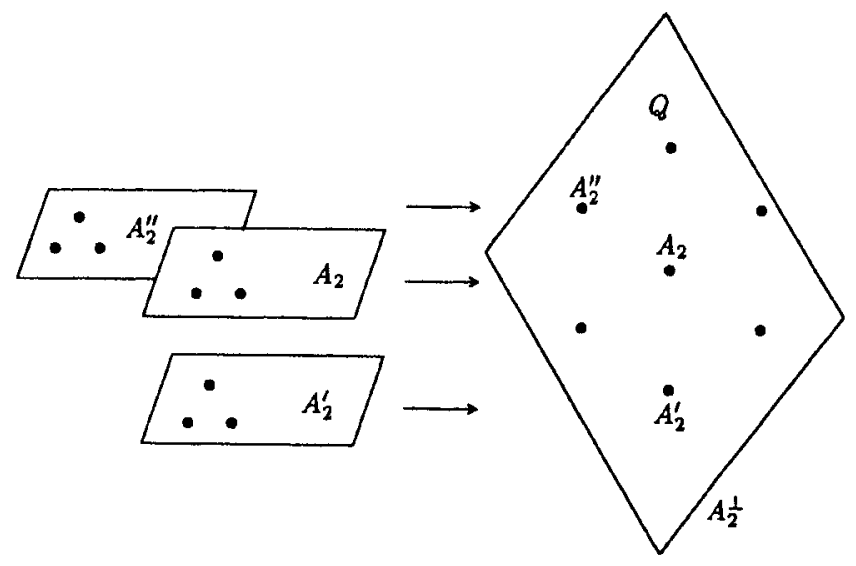

Fig. 6

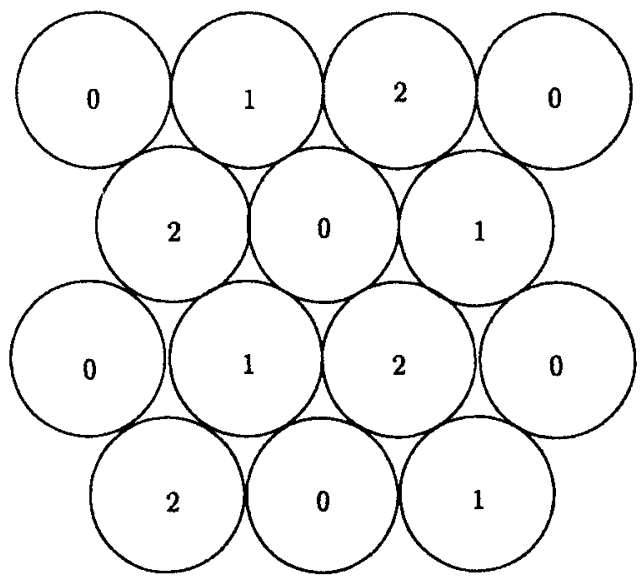

Fig. 7

\section{Dimension 5}

Proposition 5 (depends on Postulates 2, 4, and 5). The tight packings $\mathrm{II}_{5}$ are parametrized by the 4-colorings of $A_{1}$, or alternatively by random walks on a tetrahedron $4_{3}$. Just four of them are uniform packings.

Proof. The usual argument shows that any two layers must be separated by at least $\sqrt{2-1}=1$, and that if two layers $D_{4}$ and $D_{4}^{\prime}$ are separated by exactly this distance, the points in $D_{4}^{\prime}$ must lie above deep holes of $D_{4}$. In other words, $D_{4}^{\prime}$ projects into $[1] \cup[2] \cup[3]$. Once again it cannot contain two points from different cosets (whose squared distance would be an odd integer), and so must in fact be a single coset [1], [2], or [3]. So this time there are four placements [0], [1], [2], [3], forming a 
tetrahedral placement graph $4_{3}$ :

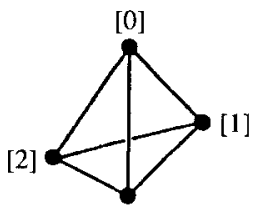

[3]

A tight five-dimensional packing $\Pi_{5}$ is specified by giving the types

$$
\cdots \quad a \quad b c d e \cdots
$$

of its layers, which constitute a 4-coloring of $A_{1}$, or a random walk on the tetrahedron $4_{3}$.

For a uniform packing there are several cases. If the neighbors of a typical sphere (say from layer $c$ ) come in antipodal pairs (this happens just when $b=d$ ) they must do so for all spheres, and we obtain the root lattice $\Lambda_{5}^{1}=\Lambda_{5}=D_{5}$, corresponding to the coloring

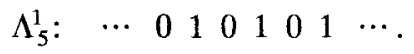

$D_{5}$ can be decomposed into such layers in several different ways.

In any other uniform packing any three adjacent letters in (1) must be distinct. Moreover, the division into layers is characteristic: adjacent spheres $A$ and $B$ are in the same layer just if there is a third sphere $C$ touching $B$ antipodally to $A$.

We now consider the spheres within the second crystal ball of a given sphere, in layer $c$ (say)-these will lie in five adjacent layers $a, b, c, d, e$, and we can ask the two questions:

$$
\text { Is } a=d ? \quad \text { Is } b=e \text { ? }
$$

The set of answers must be the same for every sphere, and determines the packing:

$$
\begin{array}{lll}
\Lambda_{5}^{2} & N N & \cdots 012301230123 \cdots \\
\Lambda_{5}^{3} & Y Y & \cdots 123123123123 \cdots \\
\Lambda_{5}^{4} & Y N & \cdots 10213203102132031 \cdots
\end{array}
$$

Thus there are four uniform packings, first found by Leech [Le1]. It turns out that $\Lambda_{5}^{n}$ consists of $n$ translates of a lattice packing ( $\left.n=1,2,3,4\right)$.

\section{Dimension 6}

Proposition 6 (depends on Postulates 2, 4, and 6). The tight packings $\mathbf{I}_{6}$ are parametrized by 4-colorings of the $A_{2}$ lattice. Just four of them are uniform.

Proof. The main assertion is proved as usual. 
Let us consider the colors of six neighbors of a circle colored $d$ in an $A_{2}$. There are essentially just four possibilities for their colors:

$a b c a b c$,

$a b a c b c$

$a b a b a b$,

ababac.

For a uniform packing, every circle in the $A_{2}$ quotient must be surrounded in the same way as every other one. It happens that each of the above four types gives a unique uniform coloring, as shown in Fig. 8, the first of them being the root lattice $\Lambda_{6}=E_{6}$. The packing $\Lambda_{6}^{n}$ consists of $n$ translates of a lattice $(n=1,2,3,4)$.
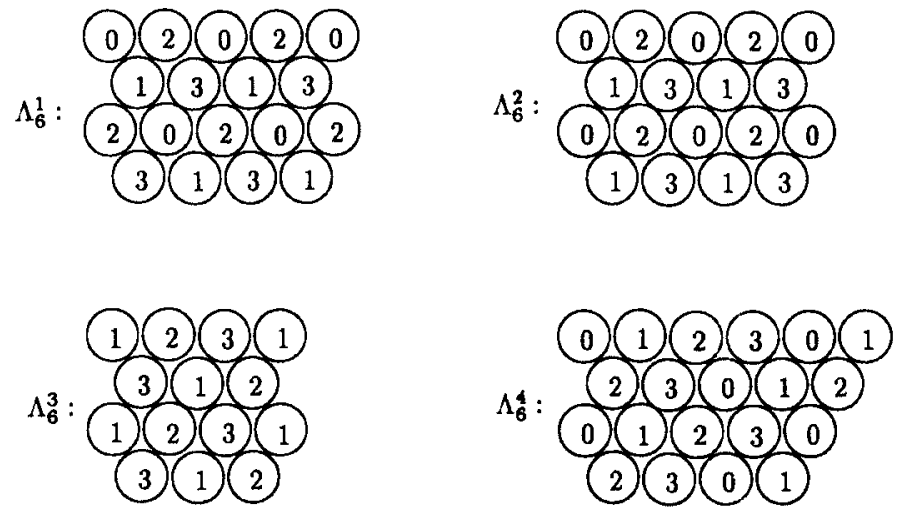

Fig. 8. The 4-colorings of $A_{2}$ that determine the four uniform tight six-dimensional packings $\Lambda_{6}^{n}$.

\section{Dimension 7}

Proposition 7 (depends on Postulates 2, 3, 4, and 7). The tight packings $\Pi_{7}$ fiber over $D_{5}$. They are parametrized by choosing a packing $\Pi_{3}$ and a "period 2 coloring" of one of its $\mathrm{A}_{2}$ layers (as defined below). Alternatively, such a packing is specified by an ordered pair consisting of a random walk on a triangle $3_{2}$ and one on a square $4_{2}$. Just four of them are uniform.

A period 2 coloring of $A_{2}$ is a 4-coloring in which the $A_{2}$ decomposes into parallel $A_{1}$ 's, each of which uses only two colors.

Proof. In the usual way we find that a $\Pi_{7}$ is determined by a $\Pi_{3}$ and a 4-coloring of it. But here a new feature occurs: there are infinitely many choices for $\Pi_{3}$.

However, in compensation, we find that the 4-coloring is completely determined by its restriction to any layer of the $\Pi_{3}$. (Each sphere of the next layer touches three spheres of this one, and so must be of the unique remaining color.) 


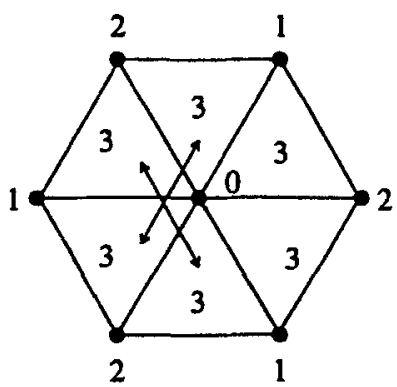

(a)

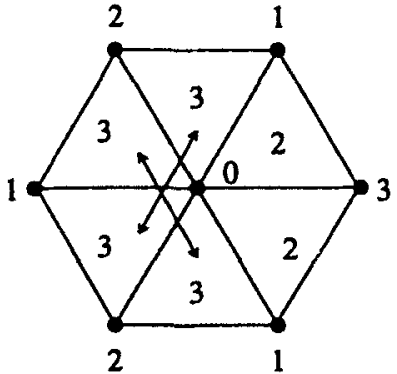

(b)

Fig. 9

This prohibits the last two cases $a b a b a b$ and $a b a b a c$ of (2), since in each case (see Fig. 9) either choice for the next layer forces two contiguous spheres to have the same color. We have therefore shown that among the six neighbors of a sphere in any layer $A_{2}$, some pair of antipodal ones have the same color, since the other two cases of (2) have this property (Fig. 10).

We next deduce that:

the $A_{2}$ can be decomposed into parallel $A_{1}$ 's, each of which uses just two colors.

For if every sphere in $A_{2}$ is surrounded as in Fig. 10(a), then the coloring has period 2 in all directions. If any one sphere is surrounded as in Fig. 11(b), then we ask: what is the pair of like-colored antipodal spheres around a sphere labeled 2 in Fig. 10(b)? The only possibility is that one of these spheres is the cental sphere colored 0 . Repeating this argument, we obtain Fig. 11, in which $a$ and $b$ must be 0 and 2 in some order, then $c$ and $d$ must be 1 and 3, and so on.

The most general $\Pi_{7}$ is therefore obtained as follows. We color one row of an $A_{2}$ lattice with two of the four colors, say 0 and 2, color the next row 1 and 3 , the next 0 and 2, and so on. We add other layers to this to obtain any of the Barlow packings, the coloring extending uniquely to these other layers. Such a packing is also

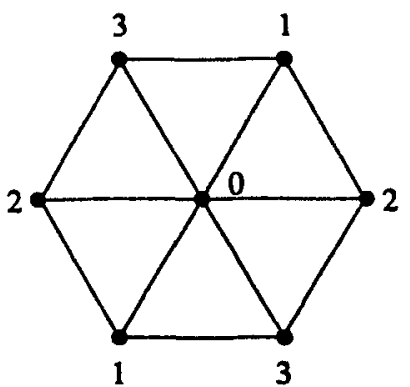

(a)

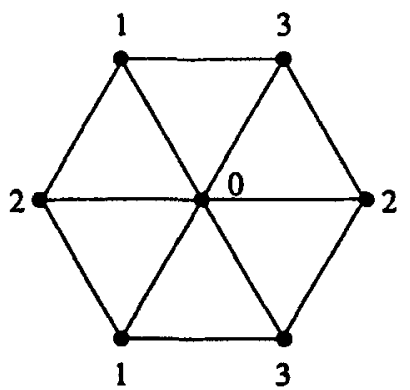

(b)

Fig. 10 


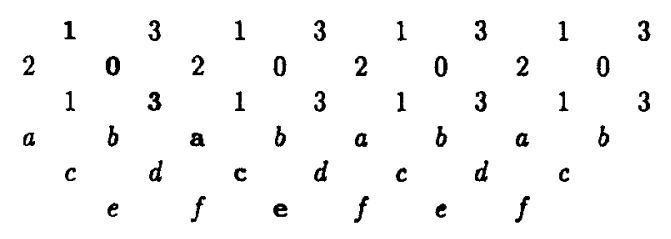

Fig. 11

completely specified by giving a random walk on the square graph

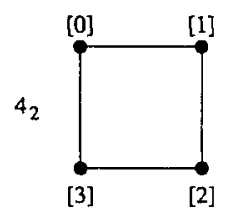

found from a sloping line such as that indicated in Fig. 11 by the bold-faced symbols, together with the random walk on the triangle $3_{2}$ that determines the associated packing $\Pi_{3}$. Since each horizontal line in our diagrams represents a translate of $D_{5}$, all such packings fiber over $D_{5}$.

There are just four cases that give uniform packings. We can extend the coloring

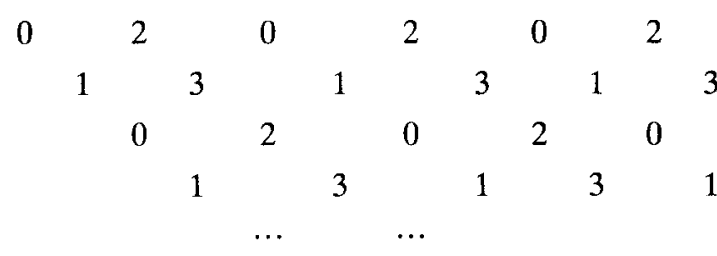

to either the f.c.c. (giving $\Lambda_{7}^{1}$ ) or the h.c.p. $\left(\Lambda_{7}^{2}\right)$, or the coloring

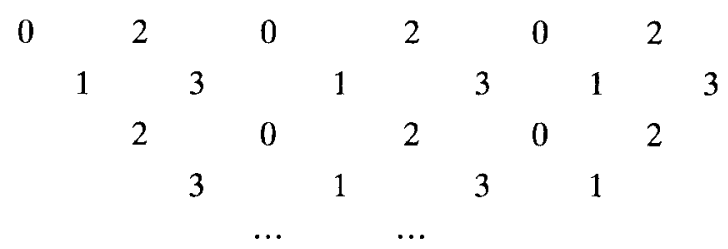

to the f.c.c. $\left(\Lambda_{7}^{3}\right)$ or the h.c.p. $\left(\Lambda_{7}^{4}\right)$. The first of these is the root lattice $\Lambda_{7}=E_{7}$, and for $n=1,2,3,4$ the packing $\Lambda_{7}^{n}$ consists of $1,2,2,4$ translates of a lattice.

\section{Dimension 8}

Proposition 8 (depends on Postulates 2, 4, and 8). The only tight packing $\Pi_{8}$ is the root lattice $\Lambda_{8}=E_{8}$.

Proof. A $\Pi_{8}$ is determined by a 4-coloring of a (rescaled) $\Pi_{4}$, equivalently of the lattice $D_{4}^{*}$. We show that this coloring is unique, and in fact assigns each vector of $D_{4}^{*}$ to the coset of $D_{4}$ it determines. 
Table 1. The unique 4-coloring of $D_{4}^{*}$.

\begin{tabular}{|c|c|c|c|c|c|c|}
\hline \multirow{2}{*}{$\frac{\text { Sphere }}{A}$} & \multicolumn{4}{|c|}{ Coordinates } & \multirow{2}{*}{$\frac{\text { Color }}{0}$} & \multirow[t]{2}{*}{ Touches } \\
\hline & 0 & 0 & 0 & 0 & & \\
\hline$B$ & $\frac{1}{2}$ & $\frac{1}{2}$ & $\frac{1}{2}$ & $\frac{1}{2}$ & 1 & $A$ \\
\hline$C$ & 0 & 0 & 0 & 1 & 2 & $A, B$ \\
\hline$D$ & $-\frac{1}{2}$ & $\frac{1}{2}$ & $\frac{1}{2}$ & $\frac{1}{2}$ & 3 & $A, B, C$ \\
\hline$E$ & 0 & 0 & 1 & 0 & 2 & $A, B, D$ \\
\hline$F$ & $-\frac{1}{2}$ & $-\frac{1}{2}$ & $\frac{1}{2}$ & $\frac{1}{2}$ & 1 & $A, C, D$ \\
\hline$G$ & $-\frac{1}{2}$ & $-\frac{1}{2}$ & $-\frac{1}{2}$ & $\frac{1}{2}$ & 3 & $A, C, F$ \\
\hline$H$ & -1 & 0 & 0 & 0 & 2 & $A, D, F$ \\
\hline$I$ & $-\frac{1}{2}$ & $-\frac{1}{2}$ & $-\frac{1}{2}$ & $-\frac{1}{2}$ & 1 & $A, G, H$ \\
\hline$J$ & -1 & -1 & 0 & 0 & 0 & $G, H, I$ \\
\hline
\end{tabular}

Consider the spheres of $D_{4}^{*}$ centered at the points shown in Table 1 . We start by arbitrarily assigning colors $0,1,2$ to the three contiguous spheres $A, B, C$. Then $D$ must be colored 3 , since it touches each of these. In a similar way we obtain the coloring of all the spheres shown in the table. It follows that this (possibly partial) coloring is invariant under permutations of the coordinates. This is because any such permutation fixes $A$ and $B$, and either fixes $C$ or takes it to another sphere like $E$ which has been assigned the same color.

The spheres mentioned in the table and their permutations show that the coloring is also invariant under changing the signs of any even number of coordinates. It is similarly invariant under subtraction of $(1,1,0,0)$. Since the images of $(1,1,0,0)$ under permutations and even sign changes generate $D_{4}$, all points of any coset of $D_{4}$ are assigned the same color, and hence the coloring and packing are unique.

\section{Dimension 9}

It is astonishing that although Blichfeldt completed the solution of the lattice version of the sphere packing problem in dimensions up to eight before 1930 (see [CS1]), the intervening 60 years have seen essentially no progress on the nine-dimensional problem. So it is only to be expected that the nonlattice problem will have new features in nine dimensions. In the next few subsections we discuss more and more surprising putatively tight packings in nine dimensions.

We first suppose the truth of Postulate 9, and also use Proposition 8, which depends on Postulates 2, 4, and 8.

\section{A. Translation}

It is known [CS1] that the deep holes in $E_{8}$ are members of the lattice $\frac{1}{2} E_{8}$. It follows that if all the layers of a $\Pi_{9}$ differ by translations, then all the corresponding placements correspond to members of $\frac{1}{2} E_{8}$. However, since shifting by a member of 
$E_{8}$ has no effect, it is better to regard the placements as members of the quotient group $\frac{1}{2} E_{8} / E_{8}$.

We recall from [CS1] the structure of $\frac{1}{2} E_{8} / E_{8}$. There are $2^{8}=256$ cosets of $E_{8}$ in $\frac{1}{2} E_{8}$, and the shortest vectors in a coset are as follows:

(i) the zero vector ( 1 coset),

(ii) $\pm \frac{1}{2} u$ (120 cosets),

(iii) $\pm \frac{1}{2} v_{1} \pm \cdots \pm \frac{1}{2} v_{8}$ (135 cosets),

where $u \in E_{8}$ is a norm 2 vector and $v_{1}, \ldots, v_{8} \in E_{8}$ are mutually orthogonal norm 4 vectors that are congruent modulo $2 E_{8}$. The deep holes in $E_{8}$ correspond to the 135 type (iii) cosets. The placement graph $256_{135}$ for this problem therefore has as nodes these 256 cosets, and two nodes are joined by an edge whenever the difference of the corresponding cosets is of type (iii). This does indeed have valence 135. Thus we have proved:

Lemma 9A. The tight packings $\mathrm{II}_{9}$ in which all layers are obtained by translations of each other are parametrized by random walks on the above graph $256_{135}$.

\section{B. Rotation}

If we allow adjacent layers to be related by rotations as well as translations there are more possibilities. To find out how many, we first consider the relationship between two adjacent layers $E$ and $F$. We suppose that $E$ is the usual $E_{8}$ defined with respect to the standard basis $e_{1}, \ldots, e_{8}$.

Each sphere of $F$ lies above a deep hole $v$ of $E$ and will touch 16 spheres of $E$. If we take $v=e_{1}=10000000$, these will be the $E$ spheres centered at

$$
v \pm e_{1}, \ldots, v \pm e_{8}
$$

and we remark that $E$ contains all the vectors

$$
z_{1} e_{1}+\cdots+z_{8} e_{8}
$$

for which the $z_{i}$ are integers with even sum, and also all vectors

$$
\pm \frac{1}{2} e_{1} \pm \cdots \pm \frac{1}{2} e_{8}
$$

for which the number of minus signs is even.

However, the relation between $E$ and $F$ is symmetrical! There will therefore be $16 F$-spheres

$$
\pm f_{1} \pm \cdots \pm f_{8}
$$

touching the $E$-sphere centered at the origin, and $F$ will contain all the vectors

$$
v+z_{1} f_{1}+\cdots+z_{8} f_{8}
$$


Table 2. All possibilities for orthonormal bases $\pm f_{1}, \ldots, \pm f_{8}$ of deep holes in $E_{8}$ containing $e_{1}{ }^{*}$

\begin{tabular}{cccc}
\hline Case & $2 f_{i}$ & Group & Index \\
\hline$d_{0}$ & $(20000000)$ & $2^{7}: S_{7}$ & $2^{0} .1=1$ \\
$d_{4}$ & $(2000) 0000$ & $2^{6}: S_{3} \times S_{4}$ & $2^{1} .35=70$ \\
& $0000(-+++)$ & & \\
$d_{6}$ & $(20) 000000$ & $2^{4}: 2^{3} S_{3}$ & $2^{3} .105=840$ \\
& $00(-+0++0)$ & & \\
$e_{7}$ & $(2) 0000000$ & $2^{7}: P S L_{3}(2)$ & $2^{6} .30=1920$ \\
& $0(-++0+00)$ & & \\
\hline
\end{tabular}

$*+$ and - denote +1 and -1 , and parentheses indicate that all cyclic permutations of the enclosed coordinates are to be applied. (The cases are named after the codes obtained by reducing their coordinates modulo 2 (see [CP]).)

for which the $z_{i}$ are integers with even sum, and also either all vectors

$$
\pm \frac{1}{2} f_{1} \pm \cdots \pm \frac{1}{2} f_{8}
$$

for which the number of minus signs is even, or all those for which this number is odd.

The vectors $\pm f_{1}, \ldots, \pm f_{8}$ must be an orthonormal basis of deep holes in $E$, one of which is the particular vector 10000000 . Up to symmetries of $E_{8}$ it turns out that there are just four possibilities for the doubled vectors $2 f_{1}, \ldots, 2 f_{8}$, as shown in Table 2. Each of these cases leads to a unique choice for $F$, since $F$ may not contain the vector $\frac{1}{2} f_{1}+\cdots+\frac{1}{2} f_{8}=\frac{1}{2} e_{1}+\cdots+\frac{1}{2} e_{8}$, which is already in $E$. We omit the arguments proving that these cases survive, and that the list is complete.

We next compute the number of possibilities for $F$. For each case the table shows the structure of the group that fixes or negates the leading vector 20000000 , and its index in the subgroup $2^{7}: S_{7}$ of all automorphisms of $E$ that do this. We conclude that the total number of such frames that contain the vector 20000000 is

$$
1+70+840+1920=2831
$$

and so the total number of choices for $F$ is

$$
2831 \cdot \frac{2160}{16}=382185
$$

since there are 2160 norm 4 vectors we could use in place of 20000000 and each of the frames contains 16 such vectors. From this we obtain:

Lemma 9B. The tight packings $\Pi_{9}$ whose layers differ by arbitrary rotations and translations are parametrized by random walks on a placement graph of form $\infty_{382185}$.

The reason there are infinitely many possible placements is really that the automorphism group of $E_{8}$ is a maximal finite subgroup of the eight-dimensional orthogonal group, and two placements may differ by a rotation not in this group. We 
can for instance find a $\Pi_{8}$ in which every pair of alternate layers is related by the matrix

$$
\frac{1}{2}\left[\begin{array}{cccccccc}
2 & 0 & 0 & 0 & 0 & 0 & 0 & 0 \\
0 & - & + & + & 0 & + & 0 & 0 \\
0 & 0 & - & + & + & 0 & + & 0 \\
0 & 0 & 0 & - & + & + & 0 & + \\
0 & + & 0 & 0 & - & + & + & 0 \\
0 & 0 & + & 0 & 0 & - & + & + \\
0 & + & 0 & + & 0 & 0 & - & + \\
0 & + & + & 0 & + & 0 & 0 & -
\end{array}\right]
$$

(corresponding to case $e_{7}$ ), whose characteristic polynomial

$$
(X-1)^{2}\left(X^{2}+\frac{3}{2} X+1\right)^{3}
$$

shows it to be of infinite order.

Since there are infinitely many placements but just 382,185 possibilities for a neighbor of a given one, the placement graph does indeed have type $\infty_{382185}$.

\section{Flotation}

It seems that of all these packings, only the lattice packing $\Lambda_{9}^{1}=\Lambda_{9}$ was previously known. This was first described by Korkine and Zolotareff [KZ] in 1873 and is their packing $T_{9}$ [CS1]. We describe it in some detail, because it can be modified in interesting ways.

$\Lambda_{9}$ consists of the vectors

$$
x_{1}, \ldots, x_{8}, 2 n \text { and } x_{1}, \ldots, x_{8}+1,2 n+1 \text {, }
$$

where $x_{1}, \ldots, x_{8}$ is a typical vector of $E_{8}$ in the standard coordinate system, and $n$ is any integer.

However, we can look at these vectors in another way! The ones with integral coordinates constitute the lattice $D_{9}$, and so

$$
\Lambda_{9}=D_{9} \cup D_{9}+\left(\left(\frac{1}{2}\right)^{8}, 0\right)
$$

This leads us to ask:

Are there other vectors $w$ for which the points of the "fluid diamond packing"

$$
D_{9}\langle w\rangle=D_{9} \cup\left(D_{9}+w\right)
$$

have minimal distance $\sqrt{2}$ ? 
Lemma 9C. The answer to the above question is "Yes." In fact there are uncountably many choices for $w$.

The condition is just that the squared distance of $w$ from the nearest point of $D_{9}$ should be at least 2 , and this is achieved for instance if any eight of the coordinates of $v$ are halves of odd integers, the ninth then being completely free. Let us write

$$
D_{9}^{\theta+}=D_{9} \cup D_{9}+\left(\left(\frac{1}{2}\right)^{8}, \frac{1}{2} \theta\right)
$$

Then $D_{9}^{0+}=\Lambda_{9}$ and $D_{9}^{1+}=D_{9}^{+}$is the nine-dimensional diamond packing [CS1].

Gold and Silver Among the Diamonds. We say that a sphere in a fluid diamond packing (3) is "golden" if its center is in $D_{9}$, and "silver" if its center is at a point of $D_{9}+w$. Then if the squared distance of $w$ from $D_{9}$ is strictly greater than 2 , no silver sphere touches any golden one. So the packing $\Lambda_{9}$ has the remarkable flotation property described in the following theorem.

Flotation Theorem. Let $X$ and $Y$ be two silver spheres in $\Lambda_{9}$. Keeping the golden spheres fuxed, the silver spheres can be collectively moved so that the final position of $X$ is the original position of $Y$. Usually the silver spheres do not touch the golden ones, and at no time do any two spheres overlap. At every stage in the motion the packing is of type $D_{9}^{\theta+}$, for $0 \leq \theta \leq 1$.

Proof. Let $\left(x_{1}, \ldots, x_{9}\right)$ and $\left(y_{1}, \ldots, y_{9}\right)$ be the centers of $X$ and $Y$. Then $x_{9}$ and $y_{9}$ are integers, but the other coordinates $x_{i}$ and $y_{i}$ are halves of odd integers. The desired motion is performed in 10 stages. In stage 0 we fix $x_{1}, \ldots, x_{8}$ and increase $x_{9}$ smoothly by $\frac{1}{2}$. Then at stage $n, 1 \leq n \leq 9$, we move the $n$th coordinate to $y_{n}$, keeping the others fixed. At all times in this motion, eight of the coordinates are halves of odd integers, and so the spheres at no time overlap. Only at the instants when some coordinate of $w$ is an integer does any silver sphere touch a golden one.

Unfortunately the existence of these "floating packings" violates our Postulate 9. We therefore simply weaken the postulate to:

Postulate $9^{*}$. Every tight nine-dimensional packing $\Pi_{9}$ either fibers over $\Pi_{8}=E_{8}$, or is a fluid diamond packing.

This is not quite so despicable as it seems, since at least Postulate $9^{*}$ entails that every tight nine-dimensional packing is isotopic to one that fibers over $\Pi_{8}$. (Two tight packings of spheres of a given radius are said to be isotopic if one can be continuously deformed into the other through tight packings of spheres of the same radius.) 
We summarize our beliefs in:

Proposition 9 (depends on Postulates 2, 4, 8, and 9*). The tight nine-dimensional packings $\Pi_{9}$ are of two kinds:

(i) Packings containing $E_{8}$, which are parametrized by random walks on the graph $\infty_{382185}$.

(ii) Fluid diamond packings $D_{9}\langle w\rangle$, parametrized by the vector $w$.

There is an uncountably infinite number of uniform packings.

Proof. Only the last assertion remains to be proved. The fluid diamond packing $D_{9}\langle w\rangle$ is seen to be uniform because it has the symmetries

$$
\begin{aligned}
& x \mapsto v+x, \\
& x \mapsto w+v-x,
\end{aligned}
$$

for every $v \in D_{9}$.

Our real reason for believing this proposition is not that we find Postulate $9^{*}$ inherently plausible (indeed, the flotation property initially made it seem extremely implausible), but rather that we have tried and failed many times to produce any other packings which are at least as good as those described there. The reader who finds our arguments unconvincing is invited to produce a putatively tight packing not covered by the proposition!

\section{Dimensions 10 and Higher}

Proposition 10 (depends on Postulates 2, 4, and 8). Postulate 10 is false, even if weakened by being asserted only up to isotopy. The tightest packing currently known is that due to Best.

Proof. The tightest packings in dimensions $8+n, 0 \leq n \leq 8$, that do fiber over $E_{8}$ are easily found by our methods. They are parametrized by what we might call $\infty_{382185}$-colorings of the tight packings $\Pi_{n}$. In other words, the "color" assigned to each sphere of $\Pi_{n}$ is a node of the placement graph $\infty_{382185}$ of Lemma $9 B$, and adjacent spheres must be colored by nodes that are adjacent in this graph. However, one such packing is the laminated lattice $\Lambda_{10}$ whose density is strictly exceeded by the 10-dimensional packing found by Best and briefly described below.

Best's packing is a uniform packing, for which we have recently given a very simple construction [CS2].

The Pentacode consists of all cyclic shifts of the four vectors

$$
\text { 01112, 03110, 21310, 21132, }
$$

and their negatives, where the digits are integers modulo 4 . (These eight words are all the words of the form $c-d, b, c, d, b+c$, where $b, c, d$ are odd.) We obtain the centers of Best's 10-dimensional packing by replacing each digit of a word of the 
pentacode by two integers according to the following scheme:

$$
\begin{aligned}
& 0 \rightarrow \text { even, even } \\
& 1 \rightarrow \text { even, odd } \\
& 2 \rightarrow \text { odd, odd } \\
& 3 \rightarrow \text { odd, even } .
\end{aligned}
$$

Our assertions about this packing follow from [CS2].

Proposition 10 suggests that an appropriate modification of Postulate 10 might be:

Postulate 10* ("Best is best"). Best's packing is the only tight packing in 10 dimensions.

The authors are of two minds about the possible truth of this postulate. We have greater confidence in:

Postulate 12*. The Coxeter-Todd lattice $\mathscr{K}_{12}$ is the only tight 12-dimensional packing.

However, we have othing plausible to offer in 11 or 13 dimensions. Neither of the packings mentioned in Postulates $10^{*}$ and $12^{*}$ fibers over $E_{8}$, and Postulates 11 and 13 fail just as dramatically. There may still be some truth in Postulates $14-16$, and so some value in understanding the packings $\Pi_{8+n}$ that $d o$ fiber over $E_{8}$.

Unfortunately the description in terms of $\infty_{382185}$-colorings of $\Pi_{n}$ does not make it clear a priori that there is more than one such $\Pi_{8+n}$. However, we remark that the graph $\infty_{382185}$, and indeed its subgraph $256_{135}$, contains a copy of the complete graph $16_{15}$ on 16 points. This is because $E_{8}$ can be embedded in a scaled copy $E_{8}^{+}$of itself having half the minimal norm [CS1]. Then $E_{8}^{+}$consists of 16 cosets of $E_{8}$, and we can take those to be the desired 16 placements, since any two of them differ by a deep hole vector. This shows us that there is a particular packing $\Pi_{8+n}$ corresponding to any 16-coloring of any tight packing $\Pi_{n}$.

We estimate that this method gives us more than $10^{7}$ distinct, putatively tight, 16-dimensional packings having the same density as the Barnes-Wall lattice $\Lambda_{16}$. This lattice $\Lambda_{16}$ is one of these packings. It corresponds to a 16-coloring of $E_{8}$ in which the colors correspond to the cosets in $E_{8}$ of a sublattice $E_{8}^{-}$that is a scaled copy of $E_{8}$ at twice the minimal norm. The automorphisms of $E_{8}$ permute these 16 colors in just $\left|16 . G L_{4}(2)\right|=8.8$ ! ways. So by applying all 16 ! color permutations we may expect to obtain at least $16 ! / 8.8 !>10^{7}$ tight packings $\Pi_{16}$.

\section{Concluding Remarks}

We have achieved what seems very likely to be a complete description of all the tight packings in up to 9 dimensions, perhaps also in 10 dimensions. However, there is little point in carrying these detailed arguments much further. In particular, it seems unprofitable to study packings that fiber over $\Pi_{16}$ 's, since $\Lambda_{16}$ is no longer unique. It may well be true that all tight packings in dimensions just above 24 fiber over the 
Leech lattice $\Lambda_{24}$. There are good reasons to believe [CS1] that there are at least 75,000 tight 25 -dimensional lattice packings, and there may well be 25 -dimensional analogues of the 9-dimensional rotation and flotation phenomena of Section 9.

\section{Acknowledgment}

We thank R. H. Hardin for some computer searches for dense packings that prompted this work.

\section{References}

[Ba1] W. Barlow, Probable nature of the internal symmetry of crystals, Nature, 29 (1883), 186-188.

[Ba2] E. S. Barnes, The construction of perfect and extreme forms II, Acta Arith., 5 (1959), 205-222.

[Be] M. R. Best, Binary codes with a minimum distance of four, IEEE Trans. Inform. Theory, 26 (1980), 738-742.

[Ch] T. W. Chaundy, The arithmetic minima of positive quadratic forms, Quart J. Math., 17 (1946), $166-192$.

[Co] J. H. Conway, Personal communication to J. Leech, 1969.

[CP] J. H. Conway and V. Pless, On the enumeration of self-dual codes, J. Combin. Theory Ser. A, 28 (1980), 26-53. (See also the corrections in J. H. Conway, V. Pless, and N. J. A. Sloane, The binary self-dual codes of length up to 32: a revised enumeration, J. Combin. Theory Ser. A, 60 (1992), 183-195.)

[CS1] J. H. Conway and N. J. A. Sloane, Sphere Packings, Lattices and Groups, 2nd edn., SpringerVerlag, New York. 1993.

[CS2] J. H. Conway and N. J. A. Sloane, Quaternary constructions for the binary single-errorcorrecting codes of Julin, Best and others, Designs Codes Cryptog., 41 (1994), 31-42.

[CT] H. S. M. Coxeter and J. A. Todd, An extreme duodenary form, Canad. J. Math., 5 (1953), 384-392.

[CR] H. M. Cundy and A. P. Rollett, Mathematical Models, Oxford University Press, Oxford, 1951.

[FT1] G. Fejes Tóth, New results in the theory of packing and covering, in Convexity and Its Applications, P. M. Gruber and J. M. Wills, editors, Birkhäuser, Basel, 1983, pp. 318-359.

[FT2] L. Fejes Tóth, Solid circle-packings and circle-coverings, Studia Sci. Math. Hungar., 3 (1968), 401-409.

[Ha1] T. C. Hales, The sphere packing problem, J. Comput. Appl. Math., 44 (1992), 41-76.

[Ha2] T. C. Hales, The status of the Kepler conjecture, Math. Intelligencer, 16(3) (1994), 47-58.

[Hs] W.YY. Hsiang, On the sphere packing problem and the proof of Kepler's conjecture, Intemat. J. Math., 4 (1993), 739-831.

[KZ] A. Korkine and G. Zolotareff, Sur les formes quadratiques, Math. Ann., 6 (1873), 366-389.

[Le1] J. Leech, Five-dimensional nonlattice sphere packings, Canad. Math. Bull., 10 (1967), 387-393.

[Le2] J. Leech, Six and seven dimensional nonlattice sphere packings, Canad. Math. Bull., 12 (1969), 151-155.

[LS1] J. Leech and N. J. A. Sloane, New sphere packings in dimensions 9-15, Bull. Amer. Math. Soc., 76 (1970), 1006-1010.

[LS2] J. Leech and N. J. A. Sloane, Sphere packing and error-correcting codes, Canad. J. Math., 23 (1971), 718-745. A revised version appears as Chapter 5 of [CS1].

[Me] S. Melmore, Densest packing of equal spheres, Nature, 159 (1947), 817.

[Mu] D. Muder, A new bound on the local density of sphere packings, Discrete Comput. Geom., 10 (1993), 351-375.

[Ro] C. A. Rogers, Packing and Covering, Cambridge University Press, Cambridge, 1964. 